Archives

$17 \mid 1996$

Hommage à Bernard Lepetit

\title{
La rue dans la pensée architecturale « moderne »
}

\section{Marilena Kourniati}

\section{(apenEdition}

\section{Journals}

Édition électronique

URL : http://journals.openedition.org/ccrh/2611

DOl : $10.4000 /$ ccrh. 2611

ISSN : $1760-7906$

Éditeur

Centre de recherches historiques - EHESS

Édition imprimée

Date de publication : 4 octobre 1996

ISSN : 0990-9141

\section{Référence électronique}

Marilena Kourniati, «La rue dans la pensée architecturale « moderne » », Les Cahiers du Centre de Recherches Historiques [En ligne], 17 | 1996, mis en ligne le 27 février 2009, consulté le 19 avril 2019. URL : http://journals.openedition.org/ccrh/2611 ; DOI : 10.4000/ccrh.2611

Ce document a été généré automatiquement le 19 avril 2019

Article L.111-1 du Code de la propriété intellectuelle. 


\title{
La rue dans la pensée architecturale «moderne »
}

\author{
Marilena Kourniati
}

Le titre « La rue dans la pensée architecturale « moderne » » indique que je me situe du côté des concepteurs-créateurs de formes spatiales et non du côté des usagers. Le déplacement, dans l'approche de mon sujet d'étude, du DEA vers la thèse, est le signe de ma rencontre avec un professeur qui, avant tout, reconnaissait aux autres le droit d'apprendre et la liberté de découvrir.

Diplômée de l'université Aristote de Thessalonique, j'arrivais à l'EHESS à Paris après des études de troisième cycle sur l'architecture urbaine (École d'architecture Paris-Belleville). Je voulais examiner la transformation de l'espace de la rue dans la pensée architecturale du mouvement moderne au $\mathrm{xx}^{\mathrm{e}}$ siècle. Architecte de formation, $\mathrm{j}$ 'ai beaucoup appris des séminaires de Bernard Lepetit car les liens perceptibles d'une société à son espace y occupaient une place centrale.

Dans le cadre du DEA « Territoires urbains » de l'EHESS, j'ai travaillé, sous la direction de Bernard Lepetit, sur la transformation de la rue, comme forme urbaine, dans la première période de l'œuvre de Le Corbusier'1.

1 Entre 1910 et 1925, la pensée de Le Corbusier sur la ville change radicalement. La nette séparation entre œuvre écrite et œuvre projetée s'exprime en trois périodes : la première, 1910-1915, est délimitée par l'écriture de l'étude Construction des villes ${ }^{2}$; la deuxième, 1914-1925, sans écrits sur la ville, est riche en œuvres projetées - neuf projets de cités ouvrières / cités-jardins - ; la troisième, 1922-1925, est caractérisée par les textes publiés dans Urbanisme, considéré aujourd'hui comme un des ouvrages les plus représentatifs de la réflexion de Le Corbusier sur la ville. Connu pour ses idées "progressistes " ${ }^{3}$, Le Corbusier commence sa réflexion sur la ville en utilisant comme référence les protagonistes du modèle culturaliste. De Construction des villes à Urbanisme, il passe de la rue perçue comme lieu d'appréhension de la ville à la rue conçue comme machine à circuler. Dépassant son rôle d'architecte-artiste ayant comme préoccupation principale 
l'esthétique de la ville, il propose de résoudre des questions urbaines urgentes, spatiales autant que sociales, en se focalisant sur les exigences des nouveaux modes de circulation.

2 J'ai d'abord examiné les propositions « opposées » (sur la rue) qui résultent de ces deux ouvrages. Ces études synchroniques essaient de fonder un discours de discipline autonome où l'urbanisme présente une pratique spécifique d'aménagement spatial ${ }^{4}$. Dans un second temps, afin d'esquisser la période transitoire entre les deux ouvrages, Construction des villes et Urbanisme, j'ai étudié son œuvre projetée. De la cité-jardin aux Cretets en 1914, extension de la ville du XIX ${ }^{e}$ siècle, Le Corbusier aboutit graduellement à la cité-jardin de 370 maisons, en 1925, extension de la ville nouvelle du $\mathrm{Xx}^{\mathrm{e}}$ siècle. J'ai procédé à l'analyse morphologique de chaque projet architectural; étude de l'organisation interne de chaque élément par décomposition de la structure physique en formes urbaines (bâti, voirie, espaces libres, parcellaire); étude de l'articulation des éléments par recomposition. Les éléments ainsi que les rapports entre eux sont décrits selon trois critères :

- topologie (par exemple : position [accolement, proximité, éloignement, franchissement] ;

liaison [continue, discontinue]),

- géométrie (par exemple : direction [interdépendance, désobéissance partielle])

- et dimensionnement ${ }^{5}$.

Le résultat de chaque rapport morphologique pour l'ensemble des projets est présenté dans des diagrammes, afin de faire apparaitre l'évolution des formes urbaines et les changements successifs de leurs rapports entre elles (du binôme maison-rue, Le Corbusier passe aux deux systèmes autonomes : réseau viaire-trame bâtie).

4 Le Corbusier annonçait, en 1912,

[l'émergence, par le] modèle de la cité-jardin, d'une vie nouvelle, à la campagne,

basée sur les valeurs mêmes de la famille.

En contrepartie de l'offre culturelle de la ville,

[l'homme] sentira la nécessité d'une culture plus domestique, plus familiale ${ }^{6}$.

Dans ses projets, la véritable «mort de la rue » n'est pas vraiment due à son sacrifice sur l'autel de la circulation mais à la disparition des espaces publics qui s'accorde avec la naissance d'une pluralité d'espaces intérieurs ${ }^{7}$.

Ce DEA a été aussi l'occasion de tester une méthode d'analyse qui met en parallèle textes et projets des architectes afin d'approcher l'univers mental des auteurs. En insistant sur la nécessité d'examiner parallèlement textes et projets, je m'efforce actuellement, dans mon travail de thèse, de décloisonner ma recherche d'une lecture interne des formes et de construire l'objet d'étude sur une série d'articulations: textes et projets, œuvre et créateur ou pratique et acteur, individu et groupe ou acteur et champ d'acteurs. Comme " terrain d'étude ", j'ai choisi un groupe d'architectes qui, au cours des années soixante et soixante-dix, se sont rassemblés sous le nom de Team X. Ce groupe développe une pensée qui se rattache directement et d'une manière critique au mouvement moderne des années "héroïques». Il tente de dépasser la pensée institutionalisée, exprimée par le CIAM (Congrès international d'architecture moderne) ${ }^{8}$, dont Le Corbusier était le protagoniste éminent. Les membres de Team X, exerçant leur métier séparément, dans différents pays d'Europe, ont constitué un groupe d'action qui développa une problématique collective pendant deux décennies 9 . Si pour Le Corbusier, dans les travaux que j'ai étudiés, le statut de la rue est ce qui fait évoluer sa conception de la ville, c'est aussi à travers des propositions du changement d'usage de la rue que vont évoluer les conceptions modernes en matière urbaine après 1945. Après la guerre, la pratique 
urbanistique est confrontée à de nouvelles demandes, à de nouveaux problèmes: reconstruction massive de logements et reconstruction des centres historiques des villes bombardées. Ainsi, dans les CIAM - après dix ans d'interruption, de 1937 à 1947 - de nouveaux thèmes émergent. Le VIII ${ }^{e}$ CIAM, en 1951, est consacré à la question

[...] d'un autre élément qui fait de la communauté une communauté et pas simplement un agrégat d'individus... ce qu'on a appelé ici the Core de la ville.

Le changement d'orientation s'exprime officiellement dans le IX ${ }^{e}$ CIAM en 1953. Son programme était d'établir la "charte d'habitat», comme prolongement de la Charte d'Athènes. C'est l'occasion pour la nouvelle génération de réévaluer les principes de l'urbanisme «moderne" et d'exprimer sa propre solution. Les grilles d'analyse présentées, comme celles du groupe anglais Mars ou du groupe Gamma travaillant sur des opérations au Maroc, mettent l'accent sur l'importance des qualités culturelles propres à chaque lieu. Le groupe Mars demande le déplacement des quatre catégories fonctionnelles-habiter, travailler, se récréer, circuler-vers la hiérarchie des « associations humaines »- habitat, rue, quartier, ville. Lors des CIAM, la rupture entre les deux générations, celle de l'avant - et celle de l'après-guerre, se cristallise pour une grande part sur une différence de conception de la rue. De la rue " machine à circuler ", les « jeunes » passent à la rue qu'ils désignent comme « association humaine ».

7 Les personnes ${ }^{10}$ à l'origine de cette réorientation, chargées d'organiser le $\mathrm{X}^{\mathrm{e}} \mathrm{CIAM}$ en 1956, forment alors Team X. Le nom CIAM (Congrès international d'architecture moderne) est remplacé par GRISP (Groupe de recherches des interrelations sociales et plastiques, nom qui souligne les nouvelles problématiques). L'impact de Team X, considéré comme la fin du mouvement moderne, va bien au-delà de l'action qu'on lui prête dans l'évolution des CIAM d'après-guerre. La réflexion de ce groupe sur les rapports entre formes physiques et rapports sociaux, notamment dans le domaine de l'espace public, me semble significative. Elle constitue à la fois une frontière et un passage entre les deux courants architecturaux-moderne et postmoderne-qui manifestent une conception diamétralement différente: de la «mort de la rue » à la prétention de sa redécouverte.

Les thèmes de ma recherche se résument donc ainsi :

- Comment les architectes de la « fin » du mouvement moderne conçoivent-ils et se représentent-ils la rue ? C'est-à-dire : comment posent-ils les problèmes, comment raisonnent-ils, comment leurs discours s'articulent-ils sous forme de textes et de projets? Il s'agit de retracer leur problématique autour de l'espace public ;

- Comment l'élaboration collective s'est-elle produite? Quel est le positionnement réciproque des personnes dans l'interaction? L'intention est de reconstituer leur univers intellectuel et leur champ d'action.

9 En quelques décennies, les personnes qui forment la «famille» Team X changent ${ }^{11}$. Leur diversité (nationalité, formation aux différents terrains d'action) constitue le premier point difficile de la recherche. Le second est leur façon particulière de fonctionner comme groupe :

Team X functions without chairman, secretary, or any bureaucratic structure... our idea was to be totally different from what had gone before... functions naturally in the manner of a real family; accepting in the beginning that when its cohesive energy died, it would died. As part of this deliberate policy no archives were kept by us... therefore documents that survive were kept for ideological reasons and or family connective reasons as one keeps mementos of emotional ties... a moth eaten leaf, some postcards, are mementos of La Sarraz ${ }^{12}$. 
10 L'intérêt se focalise sur la signification de la constitution d'un team, d'une « famille », sur l'organisation des «meetings" dans une période qui suit celle des expositions universelles et des congrès internationaux. En d'autres termes, les modalités du travail de ce champ d'acteurs, les forces constitutives de ce groupe et leur mode de fonctionnement constituent à la fois un objet d'étude et un principe d'explication.

11 Les documents des CIAM $^{13}$ d'après-guerre sont ma source initiale pour étudier la formation de cette "famille » dans les conditions héritées des CIAM, et la manière dont elle définit ces conditions dans le présent de son émergence. Les documents personnels de ses membres, en l'absence d'archives collectives, fournissent des informations pour suivre le processus de travail du groupe. Les publications de Team $\mathrm{X}^{14}$ forment une documentation supplémentaire pour examiner le discours, l'articulation exprimée entre textes et projets, et son mode de diffusion. L'appartenance de chacun aux autres modes d'action, leur pratique individuelle, peuvent nous indiquer les croisements entre eux.

Ma question est de voir comment la pratique de chacun rencontre celle des autres pour construire une pensée collective et comment, en même temps, cette problématique et cette action collective agissent sur la pratique individuelle. Il s'agit de cerner la dialectique entre pratique individuelle et action collective pour permettre d'étudier, du côté des concepteurs, les interférences entre matière et pratique : comment la pratique restitue-t-elle une forme de compréhension de l'espace pour ceux qui sont en charge d'en proposer l'aménagement?

\section{NOTES}

1. Le Corbusier, architecte d'origine suisse que l'on considère aujourd'hui comme un des «maitres » du mouvement moderne et comme la figure dominante des Congrès internationaux d'architecture moderne (CIAM). Il a publié, sous son nom, en 1943, la Charte d'Athènes, analyse fonctionnaliste de la ville établie dans le IV CIAM, en 1933 ; particulièrement actif et virulent dans ses écrits et dans ses participations à des manifestations destinées à promouvoir le mouvement.

2. L'étude Construction des villes a été écrite pour l'essentiel en 1910, par Charles-Édouard Jeanneret, qui adopte plus tard le pseudonyme de Le Corbusier. En l'absence du mot "urbanisme», Jeanneret utilise "construction». Il s'agit d'une étude commandée par son professeur Charles L'Epplatennier, directeur de l'école d'art de La Chaux-de-Fonds. Le manuscrit appartient à un collectionneur privé, C'est A. Brooks qui a prêté attention, en 1982, à cette étude longtemps méconnue par les chercheurs et c'est M. A. Emery, architecte conservateur du canton de Neuchâtel, qui l'a éditée, dans le cadre du centenaire de la ville de La Chaux-de-Fonds en 1992.

3. Dans son ouvrage, d'un impact considérable, Urbanisme, utopies et réalités, publié à Paris en 1965, pour interpréter « les idées qui fournissent ses bases à l'urbanisme » et " [en] saisir le sens réel $[. .$.$] ", Françoise Choay remonte au "pré-urbanisme» du \mathrm{XIX}^{\mathrm{e}}$ où elle distingue deux modèles-» images de la ville future » : l'un progressiste et l'autre culturaliste, qui trouvent leurs correspondances dans le discours et les propositions de l'urbanisme du xx siècle. F. Choay présente Le Corbusier en tant que représentant de l'urbanisme progressiste. Les autres figures de l'urbanisme dont les textes sont examinés dans mon mémoire sont réparties dans les deux 
modèles opposés : T. Garnier fait, selon F. Choay, partie de l'urbanisme progressiste ; C. Sitte et R. Unwin, du modèle culturaliste.

4. Camillo Sitte, Der Städtebau nach seinen küstlerischen Grundsätzen, Wien, 1889 ; Joseph Stübben, Der Städtebau, Darmstadt, 1890 ; Eugène Hénard, Études sur les transformations de Paris, Paris, 1903-1909 ; Raymond Unwin, Town-planning in Practice, London, 1909 ; Tony Garnier, Une cité industrielle : étude pour la construction des villes, Paris 1904-1917.

5. Méthode empruntée à P. Micheloni, P. Pinon, A. Borie, Formes et déformations des objets architecturaux et urbains, Paris, ENSBA, 1984, et à P. Micheloni, P. Pinon, Analyse morphologique et composition architecturale, Nanterre, APRAUS, 1987.

6. Charles-Édouard Jeanneret, Étude sur le mouvement d'art en Allemagne, La Chaux-de-Fonds, éd. Haefeli, 1912, p. 48.

7. Rues intérieures, grands escaliers, accès privés de stationnement, toits accessibles, services collectifs...

8. Les CIAM sont une des grandes manifestations constituant les points officiels d'échange des idées, des projets, de constitution des méthodes et de diffusion de leur démarche. Ils se sont tenus à intervalle régulier de 1928 à 1937 et de 1947 à 1959. Publications des CIAM : Le Corbusier, Charte d'Athènes, préf. Giraudoux, Paris, Plon, 1943 ; J.- L. Sert, Can Our Cities Survive?, Harvard, HUP, 1944 ; Grille CIAM d'urbanisme ; mise en pratique de la Charte d'Athènes, VII ${ }^{\mathrm{e}}$ CIAM, Boulogne, AA, coll. Ascoral, 1948 ; Tyrwhitt, Sert, Rogers, The Heart of the City, London, Lund Humphries, 1952 ; Newman, CIAM 1959, Otterlo, Stuttgart, Kramer, 1961 ; Publications de Siegfried Giedion, secrétaire général des CIAM et chroniqueur du mouvement. Ses archives se trouvent à l'institut GTA de Zurich.

9. Après le CIAM d'Otterlo, ils organisent des «meetings " jusqu'à la mort de Jaap Bakema en 1981.

10. J. Bakema (groupe hollandais Opbouw) ; G. Candilis et S. Woods (groupe Gamma) ; A. Van Eyck (groupe hollandais de huit) ; A. Smithson, P. Smithson, W. G. Howell, et J. Voelcker (groupe anglais Mars).

11. Au total : J. B. Bakema, G. Candilis, G. De Carlo, J. Coderch, J. De La Fuente, A. Van Eyck, R. Ersine, S. V. Ginkel, G. Grung, R. Gutmann, P. Guedes, H. J. A. Hovens Greve, W. G. Howell, C. Pologni, M. Schidhelm, P. Smithson, A. Smithson, J. Soltan, J. Voelcker, S. Woods.

12. Alison Smithson, The Emergence of Team 10 out of CIAM, (fac-similé du courrier manuscrit des membres), AA, 82, p. 4.

13. Archives CIAM, institut GTA, ETH Zurich.

14. À part la publication des documents du CIAM à Otterlo en 1959 : A. Smithson, Team X Primer 1953-1962, publié d'abord dans la revue anglaise Architectural Design, déc. 1962, puis par Studio Vista et MIT Press 1968, puis en paperback, MIT Press 1974 ; The Emergence of Team X out of CIAM, Architectural Association of Graduate School, London, 1982 ; Team X Meetings 1953-1984, Delft University Press, 1991 et Rizzoli, N. Y. 1991.

\section{AUTEUR}

\section{MARILENA KOURNIATI}

Architecte, école polytechnique de l'université Aristote, Thessalonique, 1990. CEA « Architecture urbaine », école d'architecture de Paris-Belleville, 1992. CEA « Architecture et économie des 
équipements culturels ", école d'architecture de Paris-Villemin, 1993.

EHESS, sous la direction de Bernard Lepetit : DEA « Territoires urbains », 1994.

Doctorante.

Archiviste, service de conservation des archives, Académie d'architecture de Paris. 